\title{
Empirical Study on Stock Return Volatility in China's Stock Market
}

\author{
Diao Yanhua ${ }^{1,3,}$, , Guo Siliang ${ }^{2}$ \\ ${ }^{1}$ Department of Economic Management, Shandong Xiehe University, Ji'nan, China \\ ${ }^{2}$ Department of Economics, Qilu Normal University, Ji'nan, China \\ ${ }^{3}$ Dhurakij Pundit University, Chinese International College, Bangkok, Thailand \\ Email address: \\ diaodarling@126.com (Diao Yanhua),99581735@qq.com (Diao Yanhua)
}

\section{To cite this article:}

Diao Yanhua, Guo Siliang. Empirical Study on Stock Return Volatility in China's Stock Market. Journal of Investment and Management. Vol. 4, No. 5, 2015, pp. 186-190. doi: 10.11648/j.jim.20150405.17

\begin{abstract}
Wave of financial globalization and financial innovation has brought great changes of the international financial market, the traditional measuring method is not well adapt to these new changes, this requires the presence of the new analysis method. This article will link function to copulas connect theory is introduced into the financial analysis. In this paper, the author makes an empirical analysis of Shenzhen composite index using GRCH family model, and the results show that Chinese stock yield has significant peak fat-tailed features, and have volatility clustering.
\end{abstract}

Keywords: GRCH Model, the Comprehensive Index, Volatility

\section{Introduction}

The classical capital market theory in describing the yield changes in the securities market, the econometric model generally adopted by the assumed yield variance remains the same. This model conforms to the efficient market theory in financial markets, convenient use, commonly used to predict and estimate the stock price. But on the financial data of a large number of empirical studies show that some assumptions not very reasonable. Some financial time series is often appear volatility clustering phenomenon

In order to seek the stock market price volatility behavior more accurate description and analysis, many financial experts and measurement scientists try to use a different model and the method to deal with this problem.

In 1982, Engle put forward of the ARCH (Auto regressive Conditional Heteroskedastic) model, is considered to be the most concentrated reflects the characteristic of variance changes is widely applied to financial data time series analysis model.

In recent years, domestic scholars use the ARCH model of China's stock market volatility has a lot of research results. But most are based on the Shanghai stock index as the research object to reveal the characteristics of China's stock market volatility. Domestic research focuses in the Shanghai composite index, using the ARCH model in view of the
Shenzhen index for empirical research literature is less, so this paper tries to use the ARCH model through the analysis of the Shenzhen index to reveal the characteristics of the domestic stock market volatility.

\section{The ARCH Model and Promotion}

\subsection{The Arch Model}

There is no uniform definition about the process of the ARCH. The ARCH model is in the process of research UK inflation index was proposed. The model by moving average of the past has a deadline to yield square, to depict the yield sequence of conditional heteroscedasticity properties. In 1982, Engle are defined as follows:

$$
\begin{aligned}
& \varepsilon_{t}=\sqrt{h_{t}} v_{t} \\
& v_{t} \sim i . i . d . \\
& E\left(v_{t}\right)=0, E\left(v_{t}^{2}\right)=1 \\
& h_{t}=\alpha_{0}+\sum_{j=1}^{q} \alpha_{j} \varepsilon_{t-j}^{2}
\end{aligned}
$$

$\left\{\alpha_{j}\right\}$ is unknown parameters. Meet the above process is referred to as $\mathrm{q}$ - order autoregressive conditional heteroscedastic model, notes for the ARCH (q). In order to 
ensure the conditional variance is positive, we require $\alpha_{0}>0$, $\alpha_{j} \geq 0, \mathrm{j}=1,2 \ldots \mathrm{q}$. In order to guarantee $\left\{\varepsilon_{t}^{2}\right\}$ smooth, we require $\alpha_{1}+\ldots \ldots . .+\alpha_{q}<1$. Return order number $\mathrm{q}$ determines the impact of shocks remaining length in the subsequent error variance. $\mathrm{q}$ value is larger, the longer the duration of the fluctuation. The main characteristic of this model is set random disturbance of variance under the influence of random fluctuations are past, to reflect the fluctuation of time-varying and cluster.

The model because of its clear economic meaning, and can accurately describe the characteristics of market volatility, soon became a hot issue in academic research. But the ARCH model in practice to get better fitting effect often need a larger $p$, this not only increases the be estimated the number of parameters, but also can lead to explain variables multicollinearity problem. In order to solve the problems that exist in the ARCH model, GARCH model is put forward.

\subsection{The GARCH Model}

In 1986, GARCH model is put forward by Bollerslev. And the ARCH model got the promotion. Actually GARCH model can be seen as the ARCH model of infinite order, its lagging has faster convergence than the ARCH model, and is similar to the ARMA model structure. Still represents $\left\{x_{t}\right\}$ as a random process, and it follows an- AR (1) process. The expression for the model $\operatorname{GARCH}(p, q)$ is

$$
\left\{\begin{array}{c}
x_{t}=\mu+\phi x_{t-1}+\varepsilon_{t} \\
\varepsilon_{t}=\sqrt{h_{t}} z_{t} \\
h_{t}=\alpha_{0}+\sum_{i=1}^{q} \alpha_{i} \varepsilon_{t-i}^{2}+\sum_{i=1}^{p} \beta_{i} h_{t-i} \\
z_{t} \mid I_{t-1} \sim \text { i.i.d }(0,1)
\end{array}\right.
$$

Here, $p \geq 0, q \geq 0 ; \alpha_{0}>0, \alpha_{i} \geq 0, i=1, \cdots, q ; \beta_{i} \geq 0, i=1, \cdots, p$,

Standard residual error item $z_{t}$ generally can choose a standard normal distribution, $t$ distribution or ged distribution. Because the yield of financial time series sequence generally rush thick tail characteristics, so the selection standard residual items subject to the standard normal distribution is not appropriate. We usually choose has fat-tailed distribution $\mathrm{t}$ distribution and fitting ged distribution. With characteristics of thick tail yield sequence modeling of financial time series, we generally assume that their standard residuals obey $t$ distribution, namely the GARCH - $\mathrm{t}$ model to fitting the yield distribution. Generally, $z_{t}$ are assumed to be the mean to 0 , variance of 1 regularization $t$ distribution, which is

$$
f\left(z_{t}\right)=\frac{\Gamma[(v+1) / 2]}{\Gamma(v / 2) \sqrt{\pi(v-2)}}\left[1+\frac{z_{t}^{2}}{v-2}\right]^{-\frac{v+1}{2}}
$$

Here, $v$ degrees of freedom. A large number of empirical studies have shown that GARCH $(1,1)$ model can be well fitting yield distribution of financial time series, and GARCH - $t$ model, GARCH - ged model, than GARCH - normal model fitting effect is better.

GARCH model on the basis of the ARCH model extends the conditional heteroscedasticity equation, the expression is as follows:

$$
h_{t}=\alpha_{0}+\sum_{i=1}^{q} \alpha_{i} \varepsilon_{t-i}^{2}+\sum_{j=1}^{p} \beta_{j} h_{t-j}^{2}
$$

In order to ensure the conditional variance is positive, we require $\alpha_{i}>0, \beta_{j}>0$. In order to guarantee model is smooth wide, and the presence of parameter constraints $\alpha_{1}+\ldots \ldots . .+\alpha_{q}+\beta_{1}+\ldots \ldots . .+\beta_{p}<1$.

\subsection{The EGARCH Model}

In the financial markets generally there is an asymmetric effect. That is, the bad news is bigger than the good news of fluctuations. In order to reflect the fact in 1991, Nelson began promoting the GARCH model, and he set up the EGARCH model. Compared with the formula (2), the EGARCH model of conditional variances with nonlinear form, namely

$$
\ln h_{t}=\alpha_{0}+\sum_{i=1}^{q} \frac{\left|\varepsilon_{t-i}\right|+\gamma_{i} \varepsilon_{t-i}}{\sqrt{h_{t-i}}}+\sum_{i=1}^{p} \beta_{i} \ln h_{t-i}
$$

In reality, we often want $\gamma_{i}$ is negative, so can ensure that the bad news of volatility than good news. EGARCH model can not only reflect the volatility asymmetry, and cancelled the GARCH coefficients in the process of nonnegative constraints, therefore has the strong ability of characterization of financial volatility. EGARCH model has been widely used in practice.

Here is a yield sequence, we use AR (1)-EGARCH $(1,1)$-t model to fitting the yield distribution. Model expression as follows,

$$
\left\{\begin{array}{c}
r_{t}=\mu+\phi r_{t-1}+\varepsilon_{t} \\
\varepsilon_{t}=\sqrt{h_{t}} z_{t} \\
\ln h_{t}=\alpha_{0}+\alpha_{1} \frac{\left|\varepsilon_{t-1}\right|+\gamma \varepsilon_{t-1}}{\sqrt{h_{t-1}}}+\beta_{1} \ln h_{t-1} \\
z_{t} \mid I_{t-1} \sim i . i . d(0,1)
\end{array}\right.
$$

By the formula (6) we see $r_{t} \mid I_{t-1} \sim t_{v}\left(\mu+\phi r_{t-1}, h_{t}\right)$. In practice, the standard residual error can not directly observed, so we first find out standards and residual items which is $z_{t}=\frac{\varepsilon_{t}}{\sqrt{h_{t}}}$, and assumes that the standard residual items subject $z_{t}$ to the following distribution,

$$
\sqrt{\frac{v}{v-2}} z_{t} \sim t(v)
$$

In addition, the ARCH family members including TARCH and $\mathrm{GARCH}-\mathrm{M}$ model, and so on. 


\section{The Empirical Analysis}

\subsection{Sample Data, and the Preliminary Statistical Analysis}

\subsubsection{The Sample Data}

Based on the Shenzhen stock exchange composite index as the research object, the data selection of the Shenzhen composite index of the day's closing price from January 4, 2012 to June 20, 2015 a total of 742 data. Data from the securities of the station. Stock return to the logarithmic first-order difference between two day closing index, calculation formula is:

$$
R_{t}=\ln \left(p_{t}\right)-\ln \left(p_{t-1}\right)
$$

In this paper, the data by Eviews5.0 statistical software for processing.

\subsubsection{The Description of the Sample Sequence Statistical Analysis}

Index return form new sample time series. Basic statistical analysis was carried out on the new sequence, get the return sequence diagram and descriptive analysis results.

Table 1. Basic analysis results and the sequence diagram.

\begin{tabular}{lll}
\hline The mean & standard deviation & skewness \\
\hline 0.000524 & 0.012852 & 0.445915 \\
kurtosis & JB test & P values \\
5.494873 & 275.2328 & 0.0000 \\
\hline
\end{tabular}

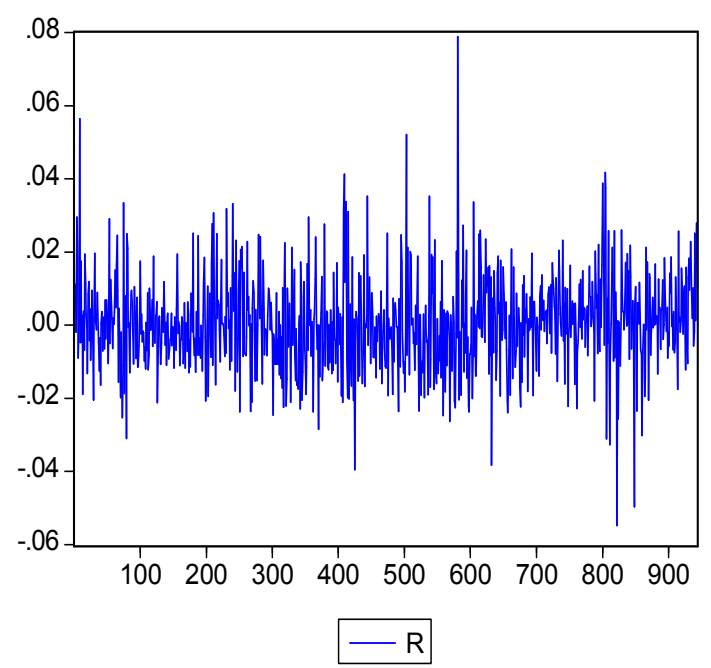

Figure 1. Basic analysis results.

Shown in table 1 , the return sequence of skewness is 0.445915 , distribution present mild right, the right of skewness distribution. That yields have a long tail, right but distribution is symmetrical; Kurtosis is 5.494873, a significant peak fat-tailed features show that sequence.

With JB statistics inspection to check whether the sequence is normal distribution, the probability value is 0 , reject the null hypothesis, that sequence does not obey the normal distribution.
Can be seen from table 1 big fluctuations followed by a significant fluctuations, the characteristics of small wave followed by the characteristics of smaller amplitude fluctuations. This shows that present day returns volatility clustering. ARCH model can well describe the characteristics of the time series.

\subsection{The Family of the Arch Model Analysis}

By judgment sample yield sequence $R_{t}$ autocorrelation function and partial autocorrelation function, we found that the yield is a three order autoregressive process. The $R_{t} \mathrm{AR}$ (3) model is established. So the estimated yield about themselves lagging of autoregressive model is:

$$
R_{t}=0.079918 R_{t-3}+\varepsilon_{t}
$$

$$
\begin{gathered}
R^{2}=0.0063 \\
\mathrm{DW}=1.9594
\end{gathered}
$$

When considering using the ARCH model for modeling, we first presence of conditional heteroscedasticity testing residual sequence. The ARCH effect of residual error sequence LM test, we found that the inspection order $q$ concomitant probability $\mathrm{p}$ are close to zero, the high order residual sequence are the ARCH effect.

Because the residual error of higher order ARCH effect, so it can build GARCH model. According to the rule of ATC and $\mathrm{SC}$, and cooperate with the residual independence test, through comparing the can get more suitable model. This article select the GARCH $(1,1)$ model, the mean equation and the conditional variance equation can be represented as:

$$
\begin{gathered}
R_{t}=0.073314 R_{t-3}+\varepsilon_{t} \\
(1.119) \\
h_{t}=0.00000703+0.059516 \varepsilon_{t-1}^{2}+0.897780 h_{t-1} \\
(2.7048)(4.344)(39.778) \\
\mathrm{AIC}=-5.91 \mathrm{SC}=-5.88
\end{gathered}
$$

We can be seen from the estimated results, parameter estimation is significant, showed that the yield sequence of $R_{t}$ has significant volatility clustering. In the conditional variance equation, coefficient and less than 1 , satisfy the constraint conditions, shows that $\operatorname{GARCH}(1,1)$ is the covariance stationary, that is, the future effects of fluctuations in the past is is gradually decline, thus the model predictability. Model of AIC and SC are small and can be thought of as good fitting the model and the data.

\subsection{TARCH $(1,1)$ Model}

In order to explain the leverage effect, we can use asymmetric ARCH model, the variance and the conditional variance equation can be expressed as: 


$$
R_{t}=\beta R_{t-3}+\varepsilon_{t} \quad h_{t}=\alpha_{0}+\alpha_{1} \varepsilon_{t-1}^{2}+\phi \varepsilon_{t-1}^{2} d_{t-1} \theta_{1} h_{t-1}
$$

Here , $d_{t}$ is a virtual variable, when $\varepsilon_{t}<0, d_{t}=$ 1 , when $\varepsilon_{t} \geq 0, d_{t}=0$. Due to the introduction of $d_{t}$, rose information $\left(\varepsilon_{t} \geq 0\right)$ and decline information $\left(\varepsilon_{t}<0\right)$ of conditional variance function is different. when rise $\phi \varepsilon_{t-1}^{2} d_{t-1}$ $=0$, we can use coefficient $\sum_{i=1}^{q} \alpha_{i}$ on behalf of its impact, when decline, we can use coefficient $\sum_{i=1}^{q} \alpha_{i}+\phi$ on behalf of its impact. If $\phi \neq 0$, means information is Asymmetric. If $\phi>0$, We believe there is leverage.

For the parameter estimation results:

$$
R_{t}=0.007220 R_{t-3}+\varepsilon_{t}
$$

$$
\begin{gathered}
h_{t}=0.00000640+0.056823 \varepsilon_{t-1}^{2}+0.904778 \varepsilon_{t-1}^{2} d_{t-1}+0.795 \theta_{1} h_{t-1} \\
(6.35)(6.58)(39.28)
\end{gathered}
$$

$$
\mathrm{AIC}=-5.93 \mathrm{SC}=-5.86
$$

Looked from the type, the model of asymmetric TARCH (1, $1), \phi$ estimate is 0.904778 . The $\mathrm{t}$ statistic value is 6.58 , significantly positive, reflecting the Chinese stock market information asymmetry and leverage.

\section{Countermeasures and Suggestions}

Price fluctuation is the basis of the normal operation of the stock market, but the risk resulting from excessive price fluctuations can cause damage to the healthy development of stock market.

The Chinese stock market is an emerging market, its history is still short, at the same time, and in the economic reform and the transformation from planned economy to market economy in the process of operation and development.

Because of the influence of the specific historical environment, on the structure specificity on the running mechanism, which makes have the features of its own on the market price risk. Compared with foreign mature stock markets, Chinese stock market on the price volatility is larger and the characteristics of explosive.

The lagging of the volatility of the stock market, yields, from another Angle shows that China's stock market prices reflect the information of sensitive enough to information exists in the transmission delay.

But in the period of time in the model can be seen that the development of China's stock market to mature gradually. China's stock market is still in the primary stage of development, at present China's securities market there are a lot of noise traders, their access to information and analysis of information ability is limited, for the performance of listed companies and the law of development ability lack of sufficient information for a long time, it was not until after the market trend to react.
Moreover, China's stock market "follow suit" phenomenon is serious, the market investors' investment and risk concept is still very immature. When the market does not have validity, when there is a great obstacles and cost information, the basis of the government intervention is reasonable. In the early stages of the development of Chinese stock market, therefore, must be a special emphasis on the establishment of the government policy on the stock market and the operation, to avoid excessive price volatility and invalid resource allocation as well as possible financial panic and crisis.

The stock market should be given to protect the interests of the investors, improve information disclosure system, ensure the equal right to obtain information, to punish insider information insider trading behavior such as false statement, prompting the rapid and accurate in the stock market information flow, promote stock market steady development and perfection.

\section{Acknowledgements}

This paper is the research findings of the present stage of Research and development projects of Shandong Province Universities(project number: J14WF69)and Humanities and Social Sciences Project of Shandong Province(project number: 14-zz-wh-04)

\section{References}

[1] Chkili Walid, Aloui Chaker, Omar Masood, John Fry. Stock market volatility and exchange rates in emerging countries: A Markov-state switching approach $[\mathrm{J}]$. Emerging Markets Review. 2011 (3)

[2] Vincent Bodart, Bertrand Candelon. Evidence of interdependence and contagion using a frequency domain framework [J]. Emerging Markets Review. 2009 (2)

[3] António Rua, Luís C. Nunes. International comovement of stock market returns: A wavelet analysis [J]. Journal of Empirical Finance. 2009 (4)

[4] Hüseyin Tastan. Estimating time-varying conditional correlations between stock and foreign exchange markets $[\mathrm{J}]$. Physica A: Statistical Mechanics and its Applications. 2005 (2)

[5] Genst. C and MacKay R. L. Copules archimédiennes et familes de lois bidimensionnelles dont lesmarges sont données [J], The Canadian Journal of Statistics, 1986, 14:145-159

[6] Joe H. Multivariate Models and Dependence Concepts [M], London: Champan \& Hall, 1997

[7] Nelsen R, An Introduction to Copulas [M], New York: Springers, 1999

[8] Frees. E. W and Valdez. E. A. Understanding Relationships using Copulas [J], North American actuarial journal, 1998, $2: 1-25$.

[9] Jing-jing jiang. Based on the GARCH - EVT - VaR model empirical research on the carbon market risk measurement $[\mathrm{J}]$. Journal of Beijing university (natural science edition), 2015, 03 
[10] Gao wei. Based on the family of GARCH model Shibor financial market volatility statistical research in China [J]. Journal of statistics and decision, 2015, 10:30-33.

\section{Biography}

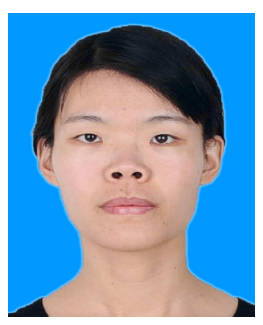

Diao Yanhua: 1983-, femail, PhD student, lecturer, mainly engaged in industrial economy, regional economy.

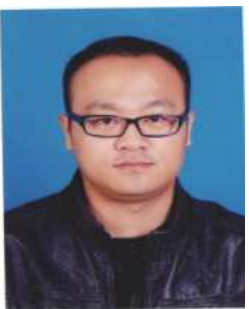

Guo Siliang: 1981-, male, lecturer, mainly engaged in technical and economic evaluation, econometrics. 